\title{
Indigenous bacterial flora of medicinal leeches and their susceptibilities to 15 antimicrobial agents
}

\author{
H. NONOMURA*,+ N. KATO $\dagger$, Y. OHNO*, M. ITOKAZU*, T. MATSUNAGA* and K. WATANABE $\dagger$ \\ *Department of Orthopaedic Surgery and + Institute of Anaerobic Bacteriology, Gifu University School of \\ Medicine, Gifu 500, Japan
}

\begin{abstract}
Surface bacterial flora, as well as homogenates, of medical leeches, Hirudo medicinalis and Hirudinaria manillensis, were surveyed and the susceptibility of these isolates to 15 antimicrobial agents was examined. Aeromonas spp. were isolated from all leeches, and Pseudomonas fuorescens and other glucose-non-fermenting gram-negative rods (NFGNR) were frequent isolates. Isolates were highly resistant to cephalosporins but susceptible to carbapenems, aminoglycosides and ofloxacin. The results indicate that prophylaxis with antimicrobial agents active against Aeromonas spp. and NF-GNR is necessary to avoid opportunist infections caused by indigenous leech flora during medical leech therapy on immunocompromised patients.
\end{abstract}

\section{Introduction}

The use of medical leeches is a useful clinical method for salvaging congested tissues after microsurgery [13]. Aeromonas hydrophila is a gram-negative aerobic organism whose habitat is mainly freshwater lakes and streams. A. hydrophila has been found to be a major member of the normal gut flora of leeches where they help to digest red blood cells, ingested in the gut lumen, because leeches themselves lack the digestive enzymes required to break down blood cells [4]. $A$. hydrophila has been implicated in various human infections such as peritonitis [5], wound infection [6], cellulitis, gastroenteritis, endocarditis, osteomyelitis, myonecrosis and sepsis [7]. This micro-organism has also been associated with infections as a complication of medical leech therapy. Lineaweaver et al. reviewed 18 clinical cases of $A$. hydrophila infection associated with the use of medical leeches [8].

Although it seems that $A$. hydrophila, as the main component of gut flora, is the sole infective agent associated with leech therapy, the possibility of local and systemic infections caused by body surface bacterial flora of leeches cannot be dismissed. So far, no study has been reported with regard to the body surface bacterial flora of medical leeches, while gut flora surveys have been conducted by several researchers.

Received 25 Jan. 1996; revised version accepted 29 April 1996.

Corresponding author: Dr H. Nonomura.
The present study attempted to isolate and identify bacteria from the body surface as well as from homogenates of medical leeches and to examine their antimicrobial susceptibilities to determine which antimicrobial agents are active against the leech flora.

\section{Materials and methods}

\section{Leeches}

Medical leeches, five specimens of Hirudo medicinalis and five of Hirudinaria manillensis, were obtained from Kono M.I. Corporation, Tokyo, Japan. These leeches were imported from the UK.

\section{Isolation of the bacterial flora of medical leeches}

The media used were Trypticase Soy Agar (BBL) supplemented with sheep blood 5\% and BTB Agar (Nissui, Tokyo, Japan) for aerobes; and Brucella HK Blood Agar (Kyokuto, Tokyo, Japan) for anaerobes. Samples were cultured in these media for $48 \mathrm{~h}$ at $37^{\circ} \mathrm{C}$ under $\mathrm{CO}_{2} 5 \%$ in air, in air or in an anaerobic atmosphere $\left(\mathrm{N}_{2} 82 \%, \mathrm{CO}_{2} 10 \%, \mathrm{H}_{2} \quad 8 \%\right)$ in an anaerobic chamber (Hirasawa, Tokyo, Japan), respectively.

Body surface bacteria of leeches were sampled by allowing 10 leeches to creep on blood agar plates for $30 \mathrm{~min}$ followed by spreading the trace with an Lshaped rod to obtain isolated colonies. Five homogenised leeches of $H$. medicinalis were used to culture total indigenous bacteria. 
For identification of bacteria, the API identification systems (bioMerieux) were used.

\section{Antibiotic susceptibility of the flora of medical leeches}

Fifteen antimicrobial agents were obtained from the following manufacturers: ampicillin, dibekacin, arbekacin, and fosfomycin (Meiji Seika Co. Ltd, Tokyo, Japan); piperacillin (Toyama Chemical Co. Ltd, Toyama, Japan); cefotiam (Takeda Chemical Industries Ltd, Tokyo, Japan); ceftazidime (Nihon Glaxo Co., Tokyo, Japan); cefmetazole and panipenem (Sankyo Co. Ltd, Tokyo, Japan); flomoxef (Shionogi and Co., Osaka, Japan); sulbactam-cefoperazone (Pfizer Pharmaceutical Inc., Tokyo, Japan); imipenem (Banyu Pharmaceuticals Co. Ltd, Tokyo, Japan); gentamicin (Schering Prau Co. Ltd, Osaka, Japan); minocycline (Lederle Japan Co. Ltd, Tokyo, Japan) and ofloxacin (Daiichi Seiyaku Co. Ltd, Tokyo, Japan). MICs were determined by the micro-broth dilution method recommended by the National Committee for Clinical Laboratory Standards [9] with cation-supplemented Mueller-Hinton II Broth (BBL).

\section{Results}

A preliminary study demonstrated a high density of body surface bacteria, isolated colonies were rarely available on the traces produced by the creeping leeches. Therefore, the traces were spread over the agar plates with an L-shaped rod to obtain isolated colonies.

Organisms cultured from the body surface and homogenates of five specimens of $H$. medicinalis and from the body surface of five $H$. manillensis are listed in Table 1.

Aeromonas spp., including A. sobria and A. hydrophila/caviae, Pseudomonas spp. and other glucosenon-fermenting gram-negative rods (NF-GNR) were isolated from the body surfaces of all leeches tested. Four of five $H$. manillensis had the Enterobacteriaceae, Citrobacter spp. and Serratia sp., while only one of five $H$. medicinalis carried the two species of enterobacteria. Two species of Staphylococcus were isolated from one $H$. medicinalis.

Aeromonas spp. were isolated from all five homogenised specimens. NF-GNR and Yersinia intermedia were isolated from two homogenised specimens.

Anaerobes were not isolated from any specimen tested on Brucella HK blood agar.

The susceptibilities of 39 bacterial isolates to 15 antimicrobial agents are given in Table 2. Ampicillin, cefotiam and fosfomycin were less active against isolates from medical leeches; the MICs of these agents were $\geqslant 32 \mu \mathrm{g} / \mathrm{ml}$ for $90 \%$ of the isolates. Piperacillin, panipenem, debekacin, arbekacin, gentamicin, minocycline and ofloxacin showed relatively good activity; $90 \%$ of the isolates were inhibited at concentrations of $\leqslant 4 \mu \mathrm{g} / \mathrm{ml}$ with these agents.

The isolates of Aeromonas spp. showed little susceptibility to ampicillin. The isolates of Ps. fluorescens and other NF-GNR were less susceptible than other species to antimicrobial agents tested.

\section{Discussion}

The gut flora of $H$. medicinalis, the traditional medical leech, have been well established. In addition to this species, H. manillensis and Asiaticobdella buntonensis were recently introduced for medical leech therapy and their gut flora were investigated. In the present study, the body surface flora of $H$. medicinalis and $H$. manillensis were surveyed, as these two leeches are now available in Japan as medical leeches.

The results show that medical leeches carry bacteria on their body surface at a high density and that Aeromonas spp. and NF-GNR (including Pseudomonas spp.) are major components in the body surface flora. While A. sobria or A. hydrophila/caviae, or both, were isolated from the homogenised specimens of all $H$. medicinalis tested, other bacteria were rarely isolated. Isolation of fewer species from homogenates than from body surfaces may be caused by an overwhelming number of Aeromonas spp. originating from the gut in the homogenates, resulting in inhibition of the growth of other bacterial species on agar media.

Previously, the gut flora of medical leeches was reported as exclusively $A$. hydrophila, with a few papers reporting low frequencies of isolation of gramnegative rods such as Pseudomonas spp. and Flavobacterium spp. [10-12].

The results of the present study, combined with previous results, suggest that Aeromonas species or $A$. hydrophila are not the only possible infectious organisms during medical leech therapy, and that Pseudomonas spp. and other NF-GNR can cause infections.

Anaerobic culture of samples for $48 \mathrm{~h}$ on Brucella HK blood agar failed to isolate any anaerobes from leeches; colonies appearing on these plates were all aerobic or facultative species. Although some plates were incubated for a further 5 days, no anaerobes were isolated, probably because of the presence of an overwhelming number of aerobic and facultative micro-organisms. These results suggest that anaerobes 
Table 1. Isolates from the homogenates and the body surfaces of five $H$. medicinalis and five H. manillensis

\begin{tabular}{|c|c|c|c|}
\hline Species & $\begin{array}{c}\text { Number of isolates from } \\
\text { the body surface of } \\
\text { H. manillensis }\end{array}$ & $\begin{array}{c}\text { Number of isolates from } \\
\text { the body surface of } \\
H . \text { medicinalis }\end{array}$ & $\begin{array}{c}\text { Number of isolates from } \\
\text { the homogenate of } \\
\text { H. medicinalis }\end{array}$ \\
\hline S. epidermidis & . & 1 & .. \\
\hline S. hominis & 1 & $\ldots$ & $\ldots$ \\
\hline Staphylococcus sp. & 1 & $\ldots$ & . . \\
\hline Micrococcus sp. & 1 & $\ldots$ & $\ldots$ \\
\hline Gram-positive coccus & $\ldots$ & $\ldots$ & 1 \\
\hline C. freundii & $\ldots$ & 1 & $\ldots$ \\
\hline Citrobacter spp. & 3 & $\ldots$ & $\ldots$ \\
\hline Pantoea (Enterobacter) agglomerans & 1 & $\ldots$ & 1 \\
\hline Serratia sp. & $\ldots$ & 1 & $\ldots$ \\
\hline Yersinia intermedia & $\ldots$ & $\ldots$ & 1 \\
\hline P. fluorescens & $\ldots$ & 2 & $\ldots$ \\
\hline P. putida & $\ldots$ & 2 & $\ldots$ \\
\hline Pseudomonas spp. & .. & 4 & $\ldots$ \\
\hline Chryseobacterium (Flavobacterium) indologens & 1 & $\ldots$ & $\ldots$ \\
\hline NF-GNR* & 5 & 6 & 5 \\
\hline A. sobria & 4 & 5 & 4 \\
\hline A. hydrophila/caviae & . . & 5 & 3 \\
\hline Aeromonas sp. & 1 & $\ldots$ & .. \\
\hline Vibrio hollisae & 1 & $\ldots$ & $\ldots$ \\
\hline Rahnella aquatilis & $\ldots$ & 1 & $\ldots$ \\
\hline Total & 19 & 28 & 15 \\
\hline
\end{tabular}

${ }^{*}$ Non-fermenting gram-negative rods.

are only a small component of the microflora in medical leeches.

Susceptibility studies demonstrated that the bacterial flora of medical leeches contained strains that were resistant to ampicillin and cephalosporins except for ceftazidime, but susceptible to carbapenems and aminoglycosides. These resistant strains included Aeromonas spp., B. fluorescens and Flavobacterium indologenes. It is notable that piperacillin was as active as ceftazidime and sulbactam-cefoperazone against isolates from the leeches tested.

Previous studies reported that $A$. hydrophila isolates were resistant to ampicillin, penicillin and firstgeneration cephalosporins, but susceptible to thirdgeneration cephalosporins, aminoglycosides, quino-

Table 2. Antimicrobial susceptibilities of 39 isolates from the body surface of $H$. medicinalis and $H$. manillensis

\begin{tabular}{lllr}
\hline & \multicolumn{3}{c}{ MIC (mg/L) } \\
\cline { 2 - 4 } Antimicrobial agent & \multicolumn{1}{c}{ Range } & $50 \%$ & $90 \%$ \\
\hline Ampicillin & $\leqq 0.125->64$ & 64 & $>64$ \\
Piperacillin & $\leqq 0.125-32$ & 2 & 4 \\
Cefotiam & $\leqq 0.125->64$ & 1 & $>64$ \\
Ceftazidime & $\leqq 0.125-32$ & 0.25 & 8 \\
Cefmetazole & $\leqq 0.125->64$ & 2 & $>64$ \\
Flomoxef & $\leqq 0.125->64$ & 1 & $>64$ \\
Sulbactam-Cefoperazone & $\leqq 0.125-16$ & 1 & 8 \\
Imipenem & $\leqq 0.125-16$ & 0.5 & 4 \\
Panipenem & $\leqq 0.125-8$ & 0.25 & 2 \\
Debekacin & $\leqq 0.125->64$ & 1 & 4 \\
Arbekacin & $\leqq 0.125-32$ & 0.5 & 2 \\
Gentamicin & $\leqq 0.125-32$ & 0.5 & 2 \\
Minocycline & $\leqq 0.125-8$ & 0.5 & 2 \\
Fosfomycin & $\leqq 0.125->64$ & 1 & $>64$ \\
Ofloxacin & $\leqq 0.125-4$ & $\leqq 0.125$ & 1 \\
\hline
\end{tabular}

lones, tetracycline and trimethoprim-sulphamethoxazole $[4,11,13]$. These results are consistent with observations in the present study, inlcuding the susceptibility of Pseudomonas spp. and other NFGNR.

Medical leeches have been shown to harbour opportunist pathogens. To avoid infection with indigenous leech flora, caution should be observed in applying medical leech therapy to immunocompromised patients. Prophylactic antibiotic therapy with thirdgeneration cephalosporins has been recommended, but it is controversial to use third-generation cephalosporins for prophylaxis, because this therapy may promote the unnecessary emergence of multi-drug resistant bacteria such as methicillin-resistant $S$. aureus. According to the results of this study, alternatives to the third-generation cephalosporins can be aminoglycosides and quinolones that are active against Aeromonas spp. and NF-GNR strains isolated from medical leeches. Another approach may be to identify bacteria from body surfaces of leeches and to perform antimicrobial susceptibility testing before starting leech therapy. In this manner, immediate prescription of proper antibiotics would be feasible when infection caused by the indigenous flora of medical leeches is suspected.

We thank G. Killgore, Centers for Disease Control and Prevention, U.S.A., for reviewing the manuscript.

\section{References}

1. Henderson HP, Matti B, Laing AG, Morelli S, Sully L. Avulsion of the scalp treated by microvascular repair: the use of leeches for post-operative decongestion. Br J Plast Surg 1983; 36: 235-239. 
2. Batchelor AG, Davison P, Sully L. The salvage of congested skin flaps by the application of leeches. Br J Plast Surg 1984; 37: $358-360$.

3. Mutimer KL, Banis JC, Upton J. Microsurgical reattachment of totally amputated ears. Plast Reconstr Surg 1987; 79: $535-541$.

4. Wells MD, Manktelow RT, Boyd JB, Bowen V. The medical leech: an old treatment revisited. Microsurgery 1993; 14: 183186.

5. Muñoz P, Fernández-Baca V, Peláez T, Sánchez R, RodriguezCréixems M, Bouza E. Aeromonas peritonitis. Clin Infect Dis 1994; 18: 32-37.

6. Blatz DJ. Open fracture of the tibia and fibula complicated by infection with Aeromonas hydrophila. A case report. $J$ Bone Joint Surg Am 1979; 61A: 790-791.

7. Janda JM, Guthertz LS, Kokka RP, Shimada T. Aeromonas species in septicemia: laboratory characteristics and clinical observations. Clin Infect Dis 1994; 19: 77-83.

8. Lineaweaver WC, Hill MK, Buncke GM et al. Aeromonas hydrophila infections following use of medicinal leeches in replantation and flap surgery. Ann Plast Surg 1992; 29: $238-244$.

9. National Committee for Clinical Laboratory Standards. Methods for dilution antimicrobial susceptibility tests for bacteria that grow aerobically M7-A2, 2nd edn. Villanova, PA. 1990.

10. Bickel KD, Lineaweaver WC, Follansbee S, Feibel R, Jackson $\mathrm{R}$, Buncke $\mathrm{HJ}$. Intestinal flora of the medicinal leech Hirudinaria manillensis. $J$ Reconstr Microsurg 1994; 10: 83-85.

11. Hermansdorfer J, Lineaweaver W, Follansbee S, Valauri FA, Buncke HJ. Antibiotic sensitivities of Aeromonas hvdrophila cultured from medicinal leeches. Br J Plast Surg 1988; 41: 649-651

12. Richerson JT, Davis JA, Meystrik R. Aeromonas, acclimation, and penicillin as complications when leeches are applied to skin flaps in rabbits. Lab Anim 1990; 24: 147-150.

13. Braga A, Lineaweaver WC, Whitney TM, Follansbee S, Buncke HJ. Sensitivities of Aeromonas hydrophila cultured from medicinal leeches to oral antibiotics. $J$ Reconstr Microsurg 1990; 6: 135-137. 